\title{
Future Small Body Exploration after the Investigation of Asteroid Itokawa by Remote Sensing and Returned Sample Analyses
}

\author{
Hajime Yano \\ Japan Aerospace Exploration Agency/Institute of Space and Aeronautical Science \\ email: yano.hajime@jaxa.jp
}

\begin{abstract}
This paper outlines current achievements of the Hayabusa mission and future small body missions with an emphasis on scientific prospects by both remote sensing in the vicinity of target objects and retuned sample analyses of them. First, the Hayabusa spacecraft aimed as technology demonstration for the worldfs first deep space round trip and sample return from an asteroid and it was launched via the M-V rocket in May of 2003. Soon after the touchdown on Asteroid Itokawa, a sub-km, S-type NEO in November 2005, the spacecraft lost its attitude control due to the leak of RCS propellant; the communication link was lost for 46 days. While the ion engine thrusters reached their lifetime by November of 2009 owing to either of an ion source or neutralizers at each engine, a challenging combination of the neutralizer-A with the ion source-B was devised to resume the spacecraftfs propulsion. This enabled the spacecraft to have returned to the Australian desert on the Earth in June 2010. The sample return capsule (SRC) was successfully recovered and returned to Japan for initial inspection of the Itokawa samples. After the announcement of initial sample analysis results, international announcement of sample distributions has started in the spring of 2012. Following up the original Hayabusa mission, JAXA has approved the Hayabusa-2 project in 2011, an asteroid sample return mission to 1999 JU3, a sub-km, C-type NEO aiming for 2014-5 launch, 2018-9 remote sensing including artificial impactor excavation and 2020 Earth return of both surface and sub-surface samples of the asteroid. C-type asteroid is thought to be abundant in organic matters and hydrated compound, so it has important clues to solve the origin and evolution of the life. NASAfs OSIRISRex and ESAfs Marco Polo-R missions are also carbonaceous asteroid sample return missions in 2010fs-2020fs. Cometary nucleus or/and D-type asteroid sample returns like Hayabusa-Mk-II concept are natural progression of this type of the endeavor. JAXAfs solar power sail mission aims for eventual rendezvous with Jovian Trojan asteroids, reservoir of D/P-type asteroids as either leftovers of Jupiter system formation or the second generation intruders from the Kuiper belt regions.
\end{abstract}

